\title{
ESTIMATIVA DA UMIDADE RELATIVA DAS 13:00 h, COM BASE NOS DADOS DAS 9:00 h E DAS 15:00 h, PARA O ESTADO DO PARANÁ
}

\author{
José Renato Soares Nunes*, Ronaldo Viana Soares**, Antônio Carlos Batista** \\ * Eng. Florestal, Dr., Depto. de Ciências Florestais (Substituto), UFPR - zerenato@floresta.ufpr.br \\ ** Eng. Florestal, Dr., Depto. de Ciências Florestais, UFPR - rvsoares@floresta.ufpr.br - batista@floresta.ufpr.br \\ Recebido para publicação: 09/05/2005 - Aceito para publicação: 06/07/2005
}

\begin{abstract}
Resumo
Estimativa da umidade relativa das 13:00 h, com base nos dados das 9:00 e das 15:00 h, para o estado do Paraná. O presente trabalho foi desenvolvido a partir de dados meteorológicos das regiões de Cambará, Campo Mourão, Cândido de Abreu, Cascavel, Guarapuava, Londrina, Palmas, Paranavaí e Pinhais, estado do Paraná. Como a Fórmula de Monte Alegre utiliza para o cálculo do índice de perigo de incêndios florestais a umidade relativa observada às 13:00 h e os padrões das estações meteorológicas da rede oficial de estações convencionais brasileiras são 9:00 e 15:00 h, buscou-se uma alternativa para a utilização do índice a partir desses dados. O objetivo consistiu em desenvolver equações que estimem os valores da Umidade Relativa às 13:00 h, com base nos dados das 9:00 e 15:00 h. Foram utilizados dados de umidade relativa das 9:00, 13:00 e 15:00 h. Os dados se referem a um período de 5 anos, entre 01/06/1998 e 31/05/2003. Foram desenvolvidas equações de regressão para cada região. As equações que apresentaram melhores resultados com base na análise dos coeficientes de determinação $\left(\mathrm{R}^{2}\right)$ e dos erros-padrão residuais $\left(\mathrm{s}_{\mathrm{y} . \mathrm{x}}\right)$ foram submetidas à análise de covariância e aquelas consideradas estatisticamente semelhantes foram combinadas, dando origem a uma equação geral que corrige a umidade relativa das 9:00 $\left(\mathrm{UR}_{09}\right)$ para as 13:00 $\mathrm{h}\left(\mathrm{UR}_{13}\right)$ e outra que corrige a umidade relativa das 15:00 $\left(\mathrm{UR}_{15}\right)$ para as 13:00 $\mathrm{h}$ para o estado do Paraná. A melhor equação para o primeiro caso foi $\mathrm{UR}_{13}=\mathrm{e}^{(2,72976+0,0162192 \mathrm{UR})}{ }_{09} \mathrm{e}$ a melhor para o segundo caso foi $\mathrm{UR}_{13}=2,451510 \mathrm{UR}_{15}{ }^{0,796072}$.

Palavras-chave: Índice de perigo; umidade relativa; meteorologia.
\end{abstract}

\section{Abstract}

Estimative of 1:00 pm relative humidity based on 9:00 am and 3:00 pm data, to Parana state, Brazil. This research was based on meteorological data from Cambará, Campo Mourão, Cândido de Abreu, Cascavel, Guarapuava, Londrina, Palmas, Paranavaí and Pinhais regions, Paraná State, Brazil. In order to use Monte Alegre Formula it was necessary to develop equations that estimate the values of Relative Humidity at 13:00 h, from the data of 9:00 and 15:00 h, standard times used in meteorological stations of the Brazilian official network. The meteorological variable used were the relative humidity data at 9:00, 13:00 and 15:00 h. The collected data refer to a period of 5 years, from June 1998 to May 2003 . Equations to estimate the 13:00 h relative humidity for each region had to be developed. Equations that presented better fits based on the determination coefficients $\left(\mathrm{R}^{2}\right)$ and the standard error of residues $\left(\mathrm{s}_{\mathrm{y} . \mathrm{x}}\right)$ were submitted to covariance analysis. Similar equations were combined, what generated a general equation that estimates relative humidity at 13:00 $\mathrm{h}\left(\mathrm{UR}_{13}\right)$ based on 9:00 $\left(\mathrm{UR}_{09}\right)$ data and estimates relative humidity at 13:00 h based on 15:00 $\left(\mathrm{UR}_{15}\right)$ data to Paraná State. The best equation in the first case was $\left.\mathrm{UR}_{13}=\mathrm{e}^{(2,72976+0,0162192 \mathrm{UR}}{ }_{09}\right)$ and in the second $\mathrm{UR}_{13}=2,451510 \mathrm{UR}_{15}{ }^{0,796072}$.

Keywords: Fire danger; relative humidity; meteorology.

\section{INTRODUÇÃO}

No estado do Paraná, a cobertura florestal vem se reduzindo ao longo das últimas décadas, seja em função da exploração comercial da matéria-prima florestal, seja pela expansão da fronteira agrícola. Aliada à crescente busca pela produção de alimento e de produtos florestais, a ocorrência de incêndios florestais vem preocupando os órgãos responsáveis pela política florestal do Estado pelos prejuízos por 
eles causados, tanto no aspecto econômico quanto no de biodiversidade. Anualmente são observadas várias ocorrências de incêndios em áreas de reflorestamento, áreas de campo nativo e em unidades de conservação sob administração estadual e federal, o que é extremamente preocupante pelos danos causados à flora, à fauna e ao solo.

A ocorrência e propagação dos incêndios florestais estão fortemente associados às condições climáticas ou fatores climáticos. A intensidade de um incêndio e a velocidade com que ele avança estão diretamente ligados à umidade relativa, temperatura e velocidade do vento. Portanto, a utilização de informações meteorológicas e climatológicas precisas é vital para o planejamento de prevenção e combate aos incêndios florestais.

Apesar do grande avanço das técnicas de combate a incêndios florestais existentes atualmente, mesmo com seus elevados custos, as técnicas de prevenção não apresentaram o mesmo ritmo de evolução. Vale ressaltar que, dentre as medidas preventivas existentes, a utilização de um índice de perigo confiável é fator fundamental para um planejamento mais eficiente das medidas de prevenção e para a adoção de ações rápidas e efetivas nas atividades de combate aos incêndios florestais, visando a redução das perdas e, conseqüentemente, dos prejuízos financeiros advindos da ocorrência de eventos catastróficos.

Outras utilidades dos índices de perigo de incêndio são o estabelecimento de zonas de risco, possibilitando um melhor planejamento das atividades de prevenção contra incêndios florestais, a permissão para queimas controladas em períodos menos perigosos, a previsão do comportamento do fogo visando a adoção de técnicas mais efetivas de combate e a advertência pública do grau de perigo, fator importantíssimo nos programas de educação ambiental, instruindo a população, através de veículos de comunicação, para que sejam adotadas medidas preventivas e, em casos extremos, limitando o acesso a áreas de risco e proibindo o uso de fogo em locais próximos à floresta.

Usada com sucesso no estado do Paraná, assim como em outras regiões do país e em alguns países da América do Sul e da África, a Fórmula de Monte Alegre (FMA) (Soares, 1972b) é um índice de perigo baseado em variáveis meteorológicas e enfatiza o aspecto da probabilidade de ignição, ou seja, indica a possibilidade de um incêndio ter início desde que haja uma fonte de fogo. Como a FMA utiliza para o cálculo do índice de perigo a umidade relativa observada às 13:00 h e o padrão das estações meteorológicas da rede oficial de estações convencionais brasileiras toma como base dos dados das 9:00 e 15:00 h, buscou-se uma alternativa para a possibilidade de utilização do índice a partir desses dados.

O objetivo deste trabalho foi desenvolver equações que estimem os valores da Umidade Relativa às 13:00 h, através dos dados das 9:00 e 15:00 h, que são os padrões utilizados nas estações meteorológicas da rede oficial brasileira, permitindo a utilização da Fórmula de Monte Alegre a partir deles.

\section{REVISÃO DE LITERATURA}

A umidade do material combustível expressa a porcentagem de água que ele contém, em relação ao seu peso seco. A primeira influência da umidade na combustão do material florestal é a necessidade da grande quantidade de energia requerida para transformar em vapor a água existente no combustível. Isso representa uma redução na quantidade de calor disponível para a combustão propriamente dita (Schroeder e Buck, 1970).

Segundo Soares (1985), o efeito da umidade do combustível na taxa de combustão do material lenhoso e na propagação dos incêndios é tão pronunciado e evidente que nenhuma medição é necessária para demonstrar sua importância, pois o material combustível com alto teor de umidade não queima. Isso se deve à denominada umidade de extinção, que é um valor do conteúdo de umidade (25 a 30\%) dos materiais combustíveis que impede a combustão e a propagação dos incêndios. $\mathrm{O}$ combustível não queima porque é necessária a utilização de uma grande quantidade de energia para vaporizar a água existente nele, ocasionando a redução da quantidade de calor disponível para a combustão propriamente dita.

Os principais fatores meteorológicos que controlam a umidade do material combustível morto são a precipitação, a umidade relativa do ar e a temperatura. Vento e radiação solar são também fatores importantes na secagem do combustível, mas eles exercem sua influência mais marcante por meio de 
mudanças na temperatura do combustível e na temperatura e umidade relativa da camada de ar próxima aos combustíveis (Schroeder e Buck, 1970).

Segundo Lowe (2001), a hora do dia tem uma influência importante nos níveis de umidade relativa. Nas primeiras horas da manhã, as temperaturas estão mais baixas e os níveis de umidade relativa mais altos. À medida que o dia passa, a temperatura começa a aumentar e a umidade relativa começa a diminuir. No meio da tarde, as temperaturas estão em seu valor máximo e a umidade relativa atinge seu valor mínimo. É importante observar periodicamente os níveis de umidade relativa, pois eles estão fortemente associados e indicam o teor de umidade dos combustíveis.

O tempo que os combustíveis florestais levam para absorver ou perder umidade para 0 ar varia bastante, dependendo do tipo e tamanho do material. Material fino, como folhas, acículas e pequenos ramos, pode alcançar um conteúdo de umidade de equilíbrio em poucos minutos. Galhos de grande dimensão e troncos podem levar um longo tempo, dependendo de sua dimensão. O tempo médio de resposta do combustível em relação à umidade do ar varia principalmente com o tamanho do material, apesar de haver outros fatores envolvidos (Batista, 1990).

A umidade atmosférica é um dos principais fatores que influem nos incêndios florestais, tendo efeito direto na inflamabilidade dos combustíveis florestais, havendo uma troca constante de umidade entre a atmosfera e os combustíveis florestais mortos. $\mathrm{O}$ material seco absorve água de uma atmosfera úmida e libera água quando o ar está seco. A quantidade de umidade que o material morto pode absorver do ar e reter depende, basicamente, da umidade relativa do ar. Durante períodos extremamente secos, a baixa umidade pode, inclusive, afetar o conteúdo de umidade do material vivo (Schroeder e Buck, 1970; Soares, 1985).

A Fórmula de Monte Alegre, desenvolvida a partir de dados da região central do estado do Paraná (Soares, 1972b), tem sido adotada em várias regiões do Brasil e da América Latina e utiliza duas variáveis: uma de forma direta, a umidade relativa do ar, medida às 13:00 horas, e outra de forma indireta, a precipitação diária.

\section{MATERIAL E MÉTODOS}

\section{Caracterização da área de estudo}

Para o desenvolvimento deste estudo foram utilizadas 9 regiões do estado do Paraná. Cada uma das regiões possui uma estação meteorológica identificada por seu código e sua posição geográfica latitude, longitude e altitude, como apresentado na Tabela 1. A Figura 1 apresenta a distribuição das regiões estudadas.

Tabela 1. Localização das estações meteorológicas nas regiões de estudo.

Table 1. Meteorological stations locations in the analyzed regions.

\begin{tabular}{|c|c|c|c|c|}
\hline Estação & Código & Latitude & Longitude & Altitude \\
\hline 1. Cambará & 23005002 & $23^{\circ} 00^{\prime} 00^{\prime \prime} \mathrm{S}$ & $50^{\circ} 02^{\prime} 00^{\prime \prime} \mathrm{W}$ & 545 \\
\hline 2. Campo Mourão & 24035222 & $24^{\circ} 03^{\prime} 00^{\prime \prime} \mathrm{S}$ & $52^{\circ} 22^{\prime} 00^{\prime \prime} \mathrm{W}$ & 630 \\
\hline 3. Cândido de Abreu & 24385115 & $24^{\circ} 38^{\prime} 00^{\prime \prime} \mathrm{S}$ & $51^{\circ} 15^{\prime} 00^{\prime}, \mathrm{W}$ & 540 \\
\hline 4. Cascavel & 24535333 & $24^{\circ} 53^{\prime} 00^{\prime \prime} \mathrm{S}$ & $53^{\circ} 33^{\prime} 00^{\prime \prime} \mathrm{W}$ & 800 \\
\hline 5. Guarapuava & 25215130 & $25^{\circ} 21^{\prime} 00{ }^{\prime} \mathrm{S}$ & $51^{\circ} 30^{\prime} 00^{\prime}, \mathrm{W}$ & 1.120 \\
\hline 6. Londrina & 23185109 & $23^{\circ} 18^{\prime} 00^{\prime \prime} \mathrm{S}$ & $51^{\circ} 09^{\prime} 00^{\prime \prime} \mathrm{W}$ & 585 \\
\hline 7. Palmas & 26285158 & $26^{\circ} 28^{\prime} 00^{\prime \prime} \mathrm{S}$ & $51^{\circ} 58^{\prime} 00^{\prime \prime} \mathrm{W}$ & 1.160 \\
\hline 8. Paranavaí & 23055226 & $23^{\circ} 05^{\prime} 00^{\prime \prime} \mathrm{S}$ & $52^{\circ} 26^{\prime} 00^{\prime \prime} \mathrm{W}$ & 470 \\
\hline 9. Pinhais & 25254905 & $25^{\circ} 25^{\prime} 00^{\prime \prime} \mathrm{S}$ & $49^{\circ} 05^{\prime} 00^{\prime}, \mathrm{W}$ & 893 \\
\hline
\end{tabular}

\section{Informações sobre o clima}

As regiões de Cambará, Campo Mourão, Cândido de Abreu, Cascavel, Londrina e Paranavaí enquadram-se no tipo Cfa, segundo a classificação de Köppen (Trewartha e Horn, 1980), caracterizado 
por possuir um clima Subtropical Úmido Mesotérmico, apresentando verões quentes (temperatura média superior a $22{ }^{\circ} \mathrm{C}$ ), invernos com geadas pouco freqüentes (temperatura média inferior a $18{ }^{\circ} \mathrm{C}$ ), sem estação seca definida.

As regiões de Guarapuava, Palmas e Pinhais enquadram-se no tipo $\mathrm{Cfb}$, caracterizado por possuir um clima Subtropical Úmido Mesotérmico, segundo a classificação de Köppen, apresentando verões frescos (temperatura média inferior a $22{ }^{\circ} \mathrm{C}$ ), invernos com ocorrências de geadas severas e freqüentes (temperatura média inferior a $18^{\circ} \mathrm{C}$ ), não apresentando estação seca.

\section{Obtenção dos dados} SIMEPAR.

A base de dados climatológicos usados neste trabalho foi fornecida pelo Instituto Tecnológico

Para cada região descrita anteriormente foram obtidos dados diários de umidade relativa às 9:00, 13:00 e 15:00 h, para um período de 5 anos, compreendido entre 01/06/1998 e 31/05/2003, totalizando 60 meses de observações.

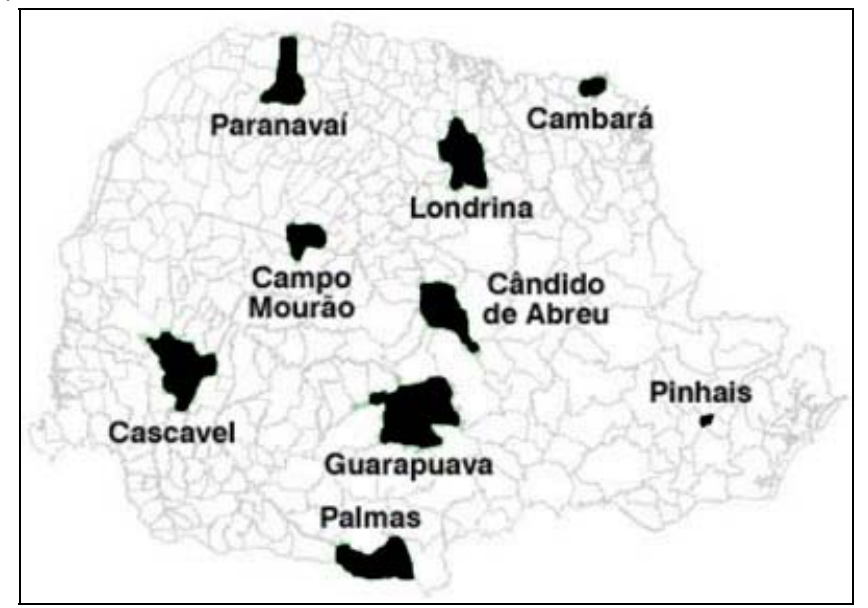

Figura 1. Distribuição das regiões de estudo no estado do Paraná.

Figure 1. Distribution of the focused regions in Parana State.

\section{Desenvolvimento de equações de regressão para umidade relativa}

Com o fim de ajustar os valores de umidade relativa das 9:00 $\mathrm{h}$ para as 13:00 $\mathrm{h}$ e das 15:00 $\mathrm{h}$ para as 13:00 h, foram geradas equações de regressão lineares, não-lineares, polinomiais e exponenciais para cada uma das regiões estudadas. As formas gerais para essas equações foram:

a) Regressão linear simples

$\mathrm{UR}_{13}=\mathrm{b}_{0}+\mathrm{b}_{1} \mathrm{UR}_{09}$

$\mathrm{UR}_{13}=\mathrm{b}_{0}+\mathrm{b}_{1} \mathrm{UR}_{15}$

b) Regressão não linear

$\mathrm{UR}_{13}=\mathrm{b}_{0} \mathrm{UR}_{09}{ }^{\mathrm{b} 1}$

$\mathrm{UR}_{13}=\mathrm{b}_{0} \mathrm{UR}_{15}{ }^{\mathrm{b} 1}$

c) Regressão polinomial

$\mathrm{UR}_{13}=\mathrm{b}_{0}+\mathrm{b}_{1} \mathrm{UR}_{09}+\mathrm{b}_{2} \mathrm{UR}_{09}^{2}$

$\mathrm{UR}_{13}=\mathrm{b}_{0}+\mathrm{b}_{1} \mathrm{UR}_{15}+\mathrm{b}_{2} \mathrm{UR}_{15}{ }^{2}$

d) Regressão exponencial

$\mathrm{UR}_{13}=\mathrm{e}^{\left(\mathrm{b}_{0}+\mathrm{b}_{1} \mathrm{UR}_{09}\right)}$

$\mathrm{UR}_{13}=\mathrm{e}^{\left(\mathrm{b}_{0}+\mathrm{b}_{1} \mathrm{UR}_{15}\right)}$

onde: $\quad \mathrm{UR}_{09}=$ umidade relativa das 9:00 $\mathrm{h}$

$\mathrm{UR}_{13}=$ umidade relativa das 13:00 $\mathrm{h}$

$\mathrm{UR}_{15}=$ umidade relativa das $15: 00 \mathrm{~h}$

$\mathrm{b}_{0}, \mathrm{~b}_{1}, \mathrm{~b}_{2}=$ coeficientes de regressão 
A seleção das equações para fornecer estimativas confiáveis para cada região baseou-se nos seguintes critérios:

a) coeficientes de determinação $\left(\mathrm{R}^{2}\right)$;

b) erros-padrão residuais $\left(\mathrm{s}_{\mathrm{y} . \mathrm{x}}\right)$.

Coeficientes de determinação maiores e menores erros-padrão residuais foram os critérios para a seleção. Ao final dessa análise foram escolhidos os melhores grupos de equações de regressão visando corrigir a umidade relativa das 9:00 h para as 13:00 h e das 15:00 h para as 13:00 h, para cada uma das regiões estudadas.

\section{Análise das equações}

Quando as equações selecionadas são do mesmo tipo, elas podem ser comparadas por meio da análise de covariância (Freese, 1967; Drapper e Smith, 1981; Nunes, 1981), tomando como base um nível de significância.

Foram realizadas análises de covariância em todas as combinações possíveis entre as nove regiões estudadas, com o fim de verificar a existência de diferença significativa entre elas ao nível de significância de 0,05 .

A análise de covariância testa, inicialmente, a significância entre as inclinações das curvas e, a seguir, as constantes de regressão, que são a interseção das curvas com o eixo Y. Se as inclinações forem estatisticamente diferentes, os grupos testados devem ter diferentes regressões e a análise deve parar nesse ponto. Se as inclinações não apresentam diferenças significativas, deve-se testar a interseção das curvas com o eixo Y. Se este teste apresentar diferença significativa, os grupos testados devem ter diferentes regressões. Se este teste não apresentar diferença significativa, as equações são consideradas semelhantes, podendo os dados ser combinados para gerar uma única equação que os represente.

\section{RESULTADOS E DISCUSSÃO}

\section{Análise da umidade relativa}

Com os dados disponíveis para todas as regiões, foram realizados testes estatísticos com o fim de se conhecer o comportamento da umidade relativa em todas as regiões estudadas.

$\mathrm{Na}$ Tabela 2 são apresentados os valores mínimos, médios e máximos diários de umidade relativa para as regiões estudadas e as variâncias e desvios-padrão correspondentes, para o período estudado.

Tabela 2. Comportamento da Umidade Relativa nas Regiões Estudadas para o Período de 01/06/1998 a 31/05/2003.

Table 2. Relative humidity characteristics in the focused regions from 06/01/1998 to 05/31/2003.

\begin{tabular}{lcccccc}
\hline \multirow{2}{*}{ Estação } & \multirow{2}{*}{$\mathbf{N}$} & \multicolumn{4}{c}{ UR às $\mathbf{1 3} \mathbf{h}(\mathbf{\%})$} & \multirow{2}{*}{$\begin{array}{c}\text { Desvio } \\
\text { padrão }\end{array}$} \\
\cline { 3 - 5 } & & Mín. & Máx. & Méd. & Variância & 16,92 \\
1. Cambará & 1826 & 19,80 & 99,70 & 60,52 & 286,25 & 16,10 \\
2. Campo Mourão & 1826 & 16,00 & 100,00 & 60,43 & 259,35 & 16,20 \\
3. Cândido de Abreu & 1826 & 18,10 & 99,80 & 61,65 & 262,36 & 18,79 \\
4. Cascavel & 1826 & 18,60 & 99,90 & 64,21 & 353,17 & 17,91 \\
5. Guarapuava & 1826 & 14,60 & 99,40 & 62,96 & 320,72 & 17,94 \\
6. Londrina & 1826 & 18,20 & 100,00 & 63,64 & 321,84 & 18,44 \\
7. Palmas & 1826 & 16,10 & 98,10 & 63,68 & 339,90 & 17,42 \\
8. Paranavaí & 1826 & 16,30 & 100,00 & 57,28 & 303,55 & 16,98 \\
9. Pinhais & 1826 & 19,80 & 100,00 & 69,74 & 288,43 & \\
\hline
\end{tabular}

As Figuras 2 e 3 apresentam, respectivamente, as umidades relativas mínimas e médias para as regiões estudadas.

Para o período estudado, observou-se que a região de Guarapuava apresentou o menor valor 
absoluto de umidade relativa (14,60\%), e as regiões de Cambará e Pinhais o maior valor para a umidade relativa mínima (19,80\%). A menor média para a umidade relativa foi de $57,28 \%$ para a região de Paranavaí, e a maior média $(69,74 \%)$ para a região de Pinhais. Esses valores estão condizentes com a classificação climática das regiões. As medidas de dispersão indicaram a região de Cascavel como tendo os maiores valores, e a região de Campo Mourão os menores valores de variância e desvio padrão.

\section{Correção da umidade relativa}

Para o cálculo da Fórmula de Monte Alegre, utiliza-se a umidade relativa das 13:00 h. Em muitos casos existem apenas os dados das 9:00 ou 15:00 h, que são os padrões utilizados nas estações meteorológicas convencionais da rede oficial brasileira, mas não existe o dado das 13:00 h. Mesmo assim, é possível a utilização da Fórmula de Monte Alegre, desde que se faça a correção dos valores da umidade relativa das 9:00 ou das 15:00 para as 13:00 h.

\section{Correção da umidade relativa das 9:00 para as 13:00 h}

As estatísticas das equações de regressão para a correção da umidade relativa das 9:00 para as 13:00 h são apresentados nas tabelas 3, 4, 5 e 6 .

Nos resultados da Tabela 3 observa-se que os coeficientes de determinação $\left(\mathrm{R}^{2}\right)$ apresentaram valores entre 0,2488 para a região de Pinhais e 0,6207 para a região de Paranavaí. Os coeficientes de correlação (r) variaram de 0,4988 a 0,7878 para as mesmas regiões, indicando uma associação moderada entre as variáveis. Os erros-padrão residuais variaram de 10,4485 para a região de Campo Mourão a 14,7987 para a região de Guarapuava.

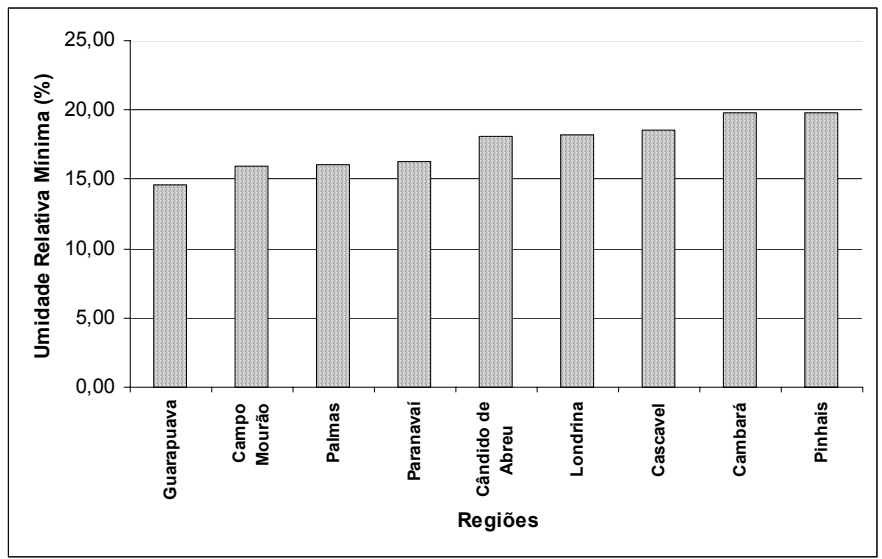

Figura 2. Umidade relativa mínima nas regiões estudadas para o período de 01/06/1998 a 31/05/2003. Figure 2. Minimum relative humidity in the focused regions from 06/01/1998 to 05/31/2003.

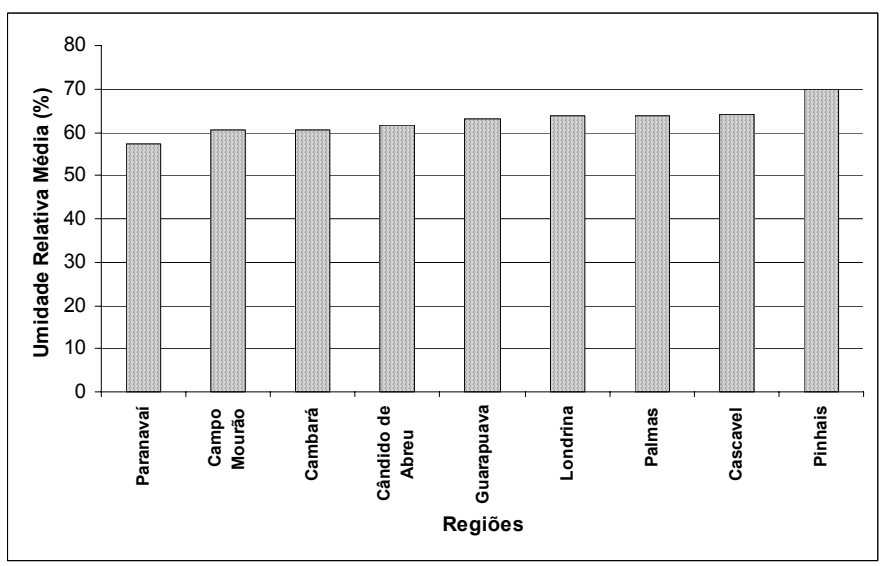

Figura 3. Umidade relativa média nas regiões estudadas para o período de 01/06/1998 a 31/05/2003. Figure 3. Average relative humidity in the focused regions from $06 / 01 / 1998$ to $05 / 31 / 2003$. 
Tabela 3. Correção da umidade relativa das 9:00 para as 13:00 h - regressão linear simples; forma geral: $\mathrm{UR}_{13}=\mathrm{b}_{0}+\mathrm{b}_{1} \mathrm{UR}_{09}$.

Table 3. Relative humidity correction from 9:00 to 13:00 $\mathrm{h}$ - simple linear regression; general model: $\mathrm{UR}_{13}=\mathrm{b}_{0}+\mathrm{b}_{1} \mathrm{UR}_{09}$.

\begin{tabular}{lcccccc}
\hline Estação & $\mathbf{N}$ & $\mathbf{b}_{\mathbf{0}}$ & $\mathbf{b}_{\mathbf{1}}$ & $\mathbf{r}$ & $\mathbf{R}^{\mathbf{2}}$ & $\mathbf{s}_{\mathbf{y} . \mathbf{x}}$ \\
\hline 1. Cambará & 1826 & $-20,293234$ & 0,914887 & 0,5925 & 0,3511 & 13,6328 \\
2. Campo Mourão & 1826 & $-20,555806$ & 1,025583 & 0,7611 & 0,5793 & 10,4485 \\
3. Cândido de Abreu & 1826 & $-16,570545$ & 0,942495 & 0,6626 & 0,4390 & 12,1346 \\
4. Cascavel & 1826 & $-18,327600$ & 1,011010 & 0,7680 & 0,5899 & 12,0661 \\
5. Guarapuava & 1826 & $-13,219934$ & 0,887998 & 0,5634 & 0,3175 & 14,7987 \\
6. Londrina & 1826 & $-32,027634$ & 1,094060 & 0,7148 & 0,5110 & 12,5479 \\
7. Palmas & 1826 & $-37,520004$ & 1,180725 & 0,6366 & 0,4053 & 14,2217 \\
8. Paranavaí & 1826 & $-13,224990$ & 0,915364 & 0,7878 & 0,6207 & 10,7328 \\
9. Pinhais & 1826 & $-11,329379$ & 0,898112 & 0,4988 & 0,2488 & 14,7235 \\
\hline
\end{tabular}

Tabela 4. Correção da umidade relativa das 9:00 para as 13:00 h - regressão não linear; forma geral: $\mathrm{UR}_{13}=\mathrm{b}_{0} \mathrm{UR}_{09} \mathrm{~b}_{1}$.

Table 4. Relative humidity correction from 9:00 to 13:00 h - non linear regression; general model: $\mathrm{UR}_{13}$ $=\mathrm{b}_{0} \mathrm{UR}_{09} \mathrm{~b}_{1}$

\begin{tabular}{lcccccc}
\hline Estação & $\mathbf{n}$ & $\mathbf{b}_{\mathbf{0}}$ & $\mathbf{b}_{\mathbf{1}}$ & $\mathbf{r}$ & $\mathbf{R}^{\mathbf{2}}$ & $\mathbf{s}_{\mathbf{y} . \mathbf{x}}$ \\
\hline 1. Cambará & 1826 & 0,145179 & 1,339900 & 0,6319 & 0,3993 & 0,2233 \\
2. Campo Mourão & 1826 & 0,163997 & 1,347970 & 0,7828 & 0,6128 & 0,1722 \\
3. Cândido de Abreu & 1826 & 0,229896 & 1,260350 & 0,6766 & 0,4579 & 0,1979 \\
4. Cascavel & 1826 & 0,207429 & 1,297680 & 0,8050 & 0,6481 & 0,1834 \\
5. Guarapuava & 1826 & 0,313047 & 1,184270 & 0,5711 & 0,3262 & 0,2492 \\
6. Londrina & 1826 & 0,077155 & 1,496090 & 0,7503 & 0,5630 & 0,1937 \\
7. Palmas & 1826 & 0,045112 & 1,621760 & 0,6456 & 0,4169 & 0,2400 \\
8. Paranavaí & 1826 & 0,318966 & 1,190120 & 0,8198 & 0,6721 & 0,1725 \\
9. Pinhais & 1826 & 0,348966 & 1,170680 & 0,5022 & 0,2522 & 0,2329 \\
\hline
\end{tabular}

Tabela 5. Correção da umidade relativa das 9:00 para as 13:00 h - regressão polinomial; forma geral: $\mathrm{UR}_{13}=\mathrm{b}_{0}+\mathrm{b}_{1} \mathrm{UR}_{09}+\mathrm{b}_{2} \mathrm{UR}_{09}^{2}$.

Table 5. Relative humidity correction from 9:00 to 13:00 h - polinomial regression; general model: $\mathrm{UR}_{13}=\mathrm{b}_{0}+\mathrm{b}_{1} \mathrm{UR}_{09}+\mathrm{b}_{2} \mathrm{UR}_{09}^{2}$.

\begin{tabular}{lccccccc}
\hline Estação & $\mathbf{N}$ & $\mathbf{b}_{\mathbf{0}}$ & $\mathbf{b}_{\mathbf{1}}$ & $\mathbf{b 2}$ & $\mathbf{R}$ & $\mathbf{R}^{\mathbf{2}}$ & $\mathbf{s}_{\mathbf{y . x}}$ \\
\hline 1. Cambará & 1826 & 47,6692 & $-0,773259$ & 0,01024 & 0,5983 & 0,3580 & 13,5633 \\
2. Campo Mourão & 1826 & 16,3872 & 0,043664 & 0,00636 & 0,7771 & 0,5856 & 10,3723 \\
3. Cândido de Abreu & 1826 & 35,7928 & $-0,383142$ & 0,00821 & 0,6671 & 0,4450 & 12,0734 \\
4. Cascavel & 1826 & 13,0368 & 0,173130 & 0,00539 & 0,7710 & 0,5945 & 12,0017 \\
5. Guarapuava & 1826 & 58,3571 & $-0,941114$ & 0,01139 & 0,5749 & 0,3305 & 14,6617 \\
6. Londrina & 1826 & 64,0806 & $-1,312850$ & 0,01469 & 0,7277 & 0,5295 & 12,3120 \\
7. Palmas & 1826 & 55,0710 & $-1,136550$ & 0,01424 & 0,6456 & 0,4168 & 14,0876 \\
8. Paranavaí & 1826 & 22,8567 & $-0,074597$ & 0,00652 & 0,7941 & 0,6306 & 10,5942 \\
9. Pinhais & 1826 & 29,8704 & $-0,109368$ & 0,00603 & 0,5007 & 0,2507 & 14,7087 \\
\hline
\end{tabular}


Nos resultados da Tabela 4, observa-se que os coeficientes de determinação $\left(\mathrm{R}^{2}\right)$ apresentaram valores entre 0,2522 para a região de Pinhais e 0,6721 para a região de Paranavaí. Os coeficientes de correlação (r) variaram de 0,5022 a 0,8198 para as mesmas regiões, indicando uma associação moderada entre as variáveis. Os erros-padrão residuais variaram de 0,1722 para a região de Campo Mourão a 0,2492 para a região de Guarapuava.

Nos resultados da Tabela 5, observa-se que os coeficientes de determinação $\left(\mathrm{R}^{2}\right)$ apresentaram valores entre 0,2507 para a região de Pinhais e 0,6306 para a região de Paranavaí. Os coeficientes de correlação (r) variaram de 0,5007 a 0,7941 para as mesmas regiões, indicando uma associação moderada entre as variáveis. Os erros-padrão residuais variaram de 10,3723 para a região de Campo Mourão a 14,7087 para a região de Pinhais.

Nos resultados da Tabela 6 , observa-se que os coeficientes de determinação $\left(\mathrm{R}^{2}\right)$ apresentaram valores entre 0,2537 para a região de Pinhais e 0,6740 para a região de Paranavaí. Os coeficientes de correlação (r) variaram de 0,5037 a 0,8210 para as mesmas regiões, indicando uma associação moderada entre as variáveis. Os erros-padrão residuais variaram de 0,1720 para a região de Paranavaí a 0,2478 para a região de Guarapuava.

O modelo de regressão exponencial teve o melhor desempenho e, portanto, será utilizado para a correção da umidade relativa das 9:00 para as 13:00 h para todas as nove regiões estudadas.

Tabela 6. Correção da umidade relativa das 9:00 para as 13:00 h - regressão exponencial; forma geral: $\mathrm{UR}_{13}=\mathrm{e}^{\left(\mathrm{b}_{0}+\mathrm{b}_{1} \mathrm{UR}_{09}\right)}$.

Table 6. Relative humidity correction from 9:00 to 13:00 $\mathrm{h}$ - exponential regression; general model:

\begin{tabular}{|c|c|c|c|c|c|c|}
\hline Estação & $\mathbf{N}$ & $\mathbf{b}_{0}$ & $\mathbf{b}_{1}$ & $\mathbf{r}$ & $\mathbf{R}^{2}$ & $S_{\mathbf{y . x}}$ \\
\hline 1. Cambará & 1826 & 2,58915 & 0,0166835 & 0,6347 & 0,4028 & 0,2226 \\
\hline 2. Campo Mourão & 1826 & 2,63712 & 0,0180793 & 0,7810 & 0,6099 & 0,1728 \\
\hline 3. Cândido de Abreu & 1826 & 2,75955 & 0,0159873 & 0,6774 & 0,4588 & 0,1978 \\
\hline 4. Cascavel & 1826 & 2,70173 & 0,0173426 & 0,8031 & 0,6450 & 0,1842 \\
\hline 5. Guarapuava & 1826 & 2,77547 & 0,0154284 & 0,5777 & 0,3337 & 0,2478 \\
\hline 6. Londrina & 1826 & 2,46080 & 0,0188826 & 0,7555 & 0,5708 & 0,1920 \\
\hline 7. Palmas & 1826 & 2,34618 & 0,0205529 & 0,6501 & 0,4226 & 0,2389 \\
\hline 8. Paranavaí & 1826 & 2,73265 & 0,0164929 & 0,8210 & 0,6740 & 0,1720 \\
\hline 9. Pinhais & 1826 & 2,91338 & 0,0143782 & 0,5037 & 0,2537 & 0,2327 \\
\hline
\end{tabular}

Correção da umidade relativa das 15:00 para as 13:00 h

As estatísticas das equações de regressão para a correção da umidade relativa das 15:00 para as 13:00 h são apresentadas nas tabelas 7, 8, 9 e 10.

Tabela 7. Correção da umidade relativa das 15:00 para as 13:00 h - regressão linear simples; forma geral: $\mathrm{UR}_{13}=\mathrm{b}_{0}+\mathrm{b}_{1} \mathrm{UR}_{15}$.

Table 7. Relative humidity correction from 15:00 to 13:00 $\mathrm{h}$ - simple linear regression; general model: $\mathrm{UR}_{13}=\mathrm{b}_{0}+\mathrm{b}_{1} \mathrm{UR}_{15}$.

\begin{tabular}{lrccccc}
\hline Estação & $\mathbf{N}$ & $\mathbf{b}_{\mathbf{0}}$ & $\mathbf{b}_{\mathbf{1}}$ & $\mathbf{r}$ & $\mathbf{R}^{\mathbf{2}}$ & $\mathbf{S}_{\mathbf{v} . \mathbf{x}}$ \\
\hline 1. Cambará & 1826 & 15,796051 & 0,824720 & 0,9107 & 0,8294 & 6,9897 \\
2. Campo Mourão & 1826 & 13,542475 & 0,826819 & 0,9274 & 0,8602 & 6,0224 \\
3. Cândido de Abreu & 1826 & 16,364563 & 0,788203 & 0,8981 & 0,8066 & 7,1242 \\
4. Cascavel & 1826 & 10,541700 & 0,882762 & 0,9311 & 0,8670 & 6,8706 \\
5. Guarapuava & 1826 & 13,801356 & 0,807103 & 0,8972 & 0,8049 & 7,9111 \\
6. Londrina & 1826 & 15,024985 & 0,834608 & 0,9295 & 0,8639 & 6,6181 \\
7. Palmas & 1826 & 11,627227 & 0,859417 & 0,9359 & 0,8759 & 6,4946 \\
8. Paranavaí & 1826 & 12,098045 & 0,847429 & 0,9149 & 0,8370 & 7,0349 \\
9. Pinhais & 1826 & 14,468765 & 0,809715 & 0,9008 & 0,8114 & 7,3766 \\
\hline
\end{tabular}


Nos resultados da Tabela 7, observa-se que os coeficientes de determinação $\left(\mathrm{R}^{2}\right)$ apresentaram valores entre 0,8114 para a região de Pinhais e 0,8759 para a região de Palmas. Os coeficientes de correlação (r) variaram de 0,90008 a 0,9359 para as mesmas regiões, indicando uma forte associação entre as variáveis. Os erros-padrão residuais variaram de 6,0224 para a região de Campo Mourão a 7,9111 para a região de Guarapuava.

Nos resultados da Tabela 8, observa-se que os coeficientes de determinação $\left(\mathrm{R}^{2}\right)$ apresentaram valores entre 0,8196 para a região de Pinhais e 0,8974 para a região de Cascavel. Os coeficientes de correlação (r) variaram de 0,9053 a 0,9473 para as mesmas regiões, indicando uma forte associação entre as variáveis. Os erros-padrão residuais variaram de 0,0945 para a região de Campo Mourão a 0,1220 para a região de Guarapuava.

Tabela 8. Correção da umidade relativa das 15:00 para as 13:00 h - regressão não linear; forma geral: $\mathrm{UR}_{13}=\mathrm{b}_{0} \mathrm{UR}_{15} \mathrm{~b}_{1}$.

Table 8. Relative humidity correction from 15:00 to 13:00 h - non linear regression; general model: $\mathrm{UR}_{13}=\mathrm{b}_{0} \mathrm{UR}_{15} \mathrm{~b}_{1}$.

\begin{tabular}{lrrrrrr}
\hline Estação & $\mathbf{n}$ & $\mathbf{b}_{\mathbf{0}}$ & $\mathbf{b}_{\mathbf{1}}$ & $\mathbf{r}$ & $\mathbf{R}^{\mathbf{2}}$ & $\mathbf{S}_{\mathbf{v} . \mathbf{x}}$ \\
\hline 1. Cambará & 1826 & 2,907430 & 0,761476 & 0,9222 & 0,8505 & 0,1114 \\
2. Campo Mourão & 1826 & 2,415780 & 0,798378 & 0,9398 & 0,8834 & 0,0945 \\
3. Cândido de Abreu & 1826 & 2,942600 & 0,751905 & 0,9100 & 0,8281 & 0,1114 \\
4. Cascavel & 1826 & 1,885150 & 0,859736 & 0,9473 & 0,8974 & 0,0990 \\
5. Guarapuava & 1826 & 2,283660 & 0,807627 & 0,9156 & 0,8384 & 0,1220 \\
6. Londrina & 1826 & 2,563460 & 0,791544 & 0,9412 & 0,8859 & 0,0990 \\
7. Palmas & 1826 & 2,056100 & 0,837442 & 0,9456 & 0,8942 & 0,1023 \\
8. Paranavaí & 1826 & 2,276050 & 0,812036 & 0,9313 & 0,8674 & 0,1097 \\
9. Pinhais & 1826 & 2,586640 & 0,780330 & 0,9053 & 0,8196 & 0,1144 \\
\hline
\end{tabular}

Nos resultados da Tabela 9, observa-se que os coeficientes de determinação $\left(\mathrm{R}^{2}\right)$ apresentaram valores entre 0,8123 para a região de Pinhais e 0,8828 para a região de Palmas. Os coeficientes de correlação (r) variaram de 0,9013 a 0,9396 para as mesmas regiões, indicando uma forte associação entre as variáveis. Os erros-padrão residuais variaram de 5,8969 para a região de Campo Mourão a 7,7069 para a região de Guarapuava.

Nos resultados da Tabela 10, observa-se que os coeficientes de determinação $\left(\mathrm{R}^{2}\right)$ apresentaram valores entre 0,7733 para a região de Cândido de Abreu e 0,8419 para a região de Cascavel. Os coeficientes de correlação (r) variaram de 0,8794 a 0,9175 para as mesmas regiões, indicando uma forte associação entre as variáveis. Os erros-padrão residuais variaram de 0,1181 para a região de Campo Mourão a 0,1343 para a região de Guarapuava.

Tabela 9. Correção da umidade relativa das 15:00 para as 13:00 h - regressão polinomial; forma geral: $\mathrm{UR}_{13}=\mathrm{b}_{0}+\mathrm{b}_{1} \mathrm{UR}_{15}+\mathrm{b}_{2} \mathrm{UR}_{15}^{2}$.

Table 9. Relative humidity correction from 15:00 to 13:00 $\mathrm{h}$ - polinomial regression; general model: $\mathrm{UR}_{13}=\mathrm{b}_{0}+\mathrm{b}_{1} \mathrm{UR}_{15}+\mathrm{b}_{2} \mathrm{UR}_{15}^{2}$.

\begin{tabular}{lccccccc}
\hline Estação & $\mathbf{n}$ & $\mathbf{b}_{\mathbf{0}}$ & $\mathbf{b}_{\mathbf{1}}$ & $\mathbf{b}_{\mathbf{2}}$ & $\mathbf{r}$ & $\mathbf{R}^{\mathbf{2}}$ & $\mathbf{s}_{\mathbf{v . x}}$ \\
\hline 1. Cambará & 1826 & 1,42248 & 1,35689 & $-0,00440341$ & 0,9175 & 0,8419 & 6.7297 \\
2. Campo Mourão & 1826 & 3,02869 & 1,20058 & $-0,00301561$ & 0,9306 & 0,8661 & 5,8969 \\
3. Cândido de Abreu & 1826 & 5,11872 & 1,18160 & $-0,00311866$ & 0,9016 & 0,8129 & 7,0099 \\
4. Cascavel & 1826 & $-2,39515$ & 1,32617 & $-0,00342707$ & 0,9345 & 0,8733 & 6,7096 \\
5. Guarapuava & 1826 & $-1,82492$ & 1,34682 & $-0,00420042$ & 0,9028 & 0,8150 & 7,7069 \\
6. Londrina & 1826 & $-0,90149$ & 1,38922 & $-0,00431941$ & 0,9358 & 0,8757 & 6,3270 \\
7. Palmas & 1826 & $-1,17005$ & 1,31209 & $-0,00359093$ & 0,9396 & 0,8828 & 6,3149 \\
8. Paranavaí & 1826 & 0,14285 & 1,28453 & $-0,00355080$ & 0,9192 & 0,8450 & 6,8633 \\
9. Pinhais & 1826 & 9,18935 & 0,98362 & $-0,00131404$ & 0,9013 & 0,8123 & 7,3612 \\
\hline
\end{tabular}


Por apresentar melhores resultados, o modelo de regressão não linear foi escolhido para realizar a correção da umidade relativa das 15:00 para as 13:00 h para todas as regiões estudadas.

\section{Análise de covariância}

Como as equações selecionadas para fazer a correção da umidade relativa das 9:00 para as 13:00 h são do mesmo tipo e as equações selecionadas para fazer a correção da umidade relativa das 15:00 para as 13:00 h são também de um mesmo tipo, cada um dos grupos pode ser comparado por meio da análise de covariância (Freese, 1967; Drapper e Smith, 1981; Nunes, 1981), tomando como base o nível de significância de 0,05 .

Foram realizadas comparações das equações selecionadas através da análise de covariância para cada região, para a correção da umidade relativa das 9:00 para as 13:00 h e das 15:00 para as 13:00 h. O resultado das análises mostrou que não houve diferença significativa em nenhuma das comparações feitas.

Duas equações são consideradas estatisticamente iguais quando os testes de significância entre as inclinações das curvas e das constantes de regressão, que são as interseções das curvas com o eixo Y, apresentam resultados estatisticamente semelhantes, ou seja, não apresentam diferença significativa para o nível de significância tomado como base. Como todas as comparações apresentaram resultados não significativos, as novas equações foram ajustadas com base nos valores combinados de todas as regiões.

\section{Correção da umidade relativa das 9:00 e das 15:00 para as 13:00 h com os dados combinados}

Com os dados combinados das nove regiões, foram geradas as equações globais para o estado do Paraná, para a correção da umidade relativa das 9:00 e das 15:00 para as 13:00 h.

Para o primeiro caso, correção da umidade relativa das 9:00 para as 13:00 h, foi gerada a equação segundo o modelo de regressão exponencial, cujos coeficientes são apresentados na Tabela 11.

Tabela 10. Correção da umidade relativa das 15:00 para as 13:00 h - regressão exponencial; forma geral: $\mathrm{UR}_{13}=\mathrm{e}^{\left(\mathrm{b}_{0}+\mathrm{b}_{1} \mathrm{UR}_{15}\right)}$.

Table 10. Relative humidity correction from 15:00 to 13:00 h-exponential regression; general model: $\mathrm{UR}_{13}=\mathrm{e}^{\left(\mathrm{b}_{0}+\mathrm{b}_{1} \mathrm{UR}_{15}\right)}$.

\begin{tabular}{lcccccc}
\hline Estação & $\mathbf{n}$ & $\mathbf{b}_{\mathbf{0}}$ & $\mathbf{b}_{\mathbf{1}}$ & $\mathbf{r}$ & $\mathbf{R}^{\mathbf{2}}$ & $\mathbf{S}_{\mathbf{v . x}}$ \\
\hline 1. Cambará & 1826 & 3,32104 & 0,013678 & 0,8873 & 0,7873 & 0,1328 \\
2. Campo Mourão & 1826 & 3,27932 & 0,013850 & 0,9044 & 0,8179 & 0,1181 \\
3. Cândido de Abreu & 1826 & 3,35067 & 0,012805 & 0,8794 & 0,7733 & 0,1279 \\
4. Cascavel & 1826 & 3,24994 & 0,014270 & 0,9175 & 0,8419 & 0,1229 \\
5. Guarapuava & 1826 & 3,28216 & 0,013411 & 0,8797 & 0,7739 & 0,1443 \\
6. Londrina & 1826 & 3,33856 & 0,013277 & 0,9055 & 0,8198 & 0,1244 \\
7. Palmas & 1826 & 3,24214 & 0,014292 & 0,9131 & 0,8337 & 0,1282 \\
8. Paranavaí & 1826 & 3,23865 & 0,014335 & 0,8951 & 0,8012 & 0,1343 \\
9. Pinhais & 1826 & 3,34757 & 0,012652 & 0,8879 & 0,7883 & 0,1239 \\
\hline
\end{tabular}

Tabela 11. Correção da umidade relativa das 9:00 para as 13:00 h para o estado do Paraná - regressão exponencial; forma geral: $\mathrm{UR}_{13}=\mathrm{e}^{\left(\mathrm{b}_{0}+\mathrm{b}_{1} \cdot \mathrm{UR}_{09}\right)}$.

Table 11. Relative humidity correction from 9:00 to 13:00 h to Paraná State - exponential regression; general model: $\mathrm{UR}_{\mathbf{1 3}}=\mathrm{e}^{\left(\mathrm{b}_{0}+\mathrm{b}_{1} \mathrm{UR}_{09}\right)}$.

\begin{tabular}{lcccccc}
\hline Estação & $\mathbf{n}$ & $\mathbf{b}_{0}$ & $\mathbf{b}_{1}$ & $\mathbf{r}$ & $\mathbf{R}^{\mathbf{2}}$ & $\mathbf{s}_{\mathbf{y . x}}$ \\
\hline Todas & 16434 & 2,72976 & 0,0162192 & 0,6898 & 0,4759 & 0,2147 \\
\hline
\end{tabular}

Observa-se que o coeficiente de determinação $\left(\mathrm{R}^{2}\right)$ foi de 0,4759 e o coeficiente de correlação $(\mathrm{r})$ de 0,6898, o que indica uma associação moderada entre as variáveis. O erro padrão residual foi de 0,2147.

A equação para realizar a correção da umidade relativa das 9:00 para as 13:00 h para o estado do Paraná tem o seguinte formato: $\mathrm{UR}_{13}=\mathrm{e}^{\left(2,72976+0,0162192 \mathrm{UR}_{09}\right)}$. 
Para o segundo caso, correção da umidade relativa das 15:00 para as 13:00 h, foi gerada a equação segundo o modelo de regressão não linear, cujos coeficientes são apresentados na Tabela 12.

Observa-se que o coeficiente de determinação $\left(\mathrm{R}^{2}\right)$ foi de 0,8639 e o coeficiente de correlação (r) foi de 0,9295, o que indica uma forte associação entre as variáveis. O erro padrão residual foi de 0,1094.

A equação para realizar a correção da umidade relativa das 15:00 para as 13:00 h para o estado do Paraná tem o seguinte formato: $\mathrm{UR}_{13}=2,451510$. UR $\mathrm{UR}_{15}^{0,796072}$.

Tabela 12. Correção da umidade relativa das 15:00 para as 13:00 h para o estado do Paraná - regressão não linear; forma geral: $\mathrm{UR}_{13}=\mathrm{b}_{0} \mathrm{UR}_{15} \mathrm{~b}_{1}$.

Table 12. Relative humidity correction from 15:00 to 13:00 h to Paraná State - non linear regression; general model: $\mathrm{UR}_{13}=\mathrm{b}_{0} \mathrm{UR}_{15}{ }^{\mathrm{b}} 1$.

\begin{tabular}{lcccccc}
\hline Estação & $\mathbf{n}$ & $\mathbf{b}_{0}$ & $\mathbf{B}_{1}$ & $\mathbf{r}$ & $\mathbf{R}^{\mathbf{2}}$ & $\mathbf{s}_{\mathbf{y . x}}$ \\
\hline Todas & 16434 & 2,451510 & 0,796072 & 0,9295 & 0,8639 & 0,1094 \\
\hline
\end{tabular}

\section{CONCLUSÕES}

Com base nos estudos e análises realizados neste trabalho, pode-se concluir que:

- a estimativa da umidade relativa diária das 13:00 h, para o estado do Paraná, pode ser obtida através da leitura da umidade relativa das 9:00 h, mediante a equação $\mathrm{UR}_{13}=\mathrm{e}^{(2,72976+0,0162192 \text { UR09); }}$

- por não apresentar alto coeficiente de determinação $\left(\mathrm{R}^{2}=0,4759\right)$ entre as variáveis $U_{13} \mathrm{e}$ $\mathrm{UR}_{09}$, a correção da umidade relativa das 9:00 para as 13:00 h deve ser usada com restrições;

- a estimativa da umidade relativa diária das 13:00 h, para o estado do Paraná, pode ser obtida através da leitura da umidade relativa das $15: 00 \mathrm{~h}$, mediante a equação $\mathrm{UR}_{13}=2,451510 \mathrm{UR}_{15}{ }^{0,796072}$;

- por apresentar alto coeficiente de determinação $\left(\mathrm{R}^{2}=0,8639\right)$ entre as variáveis $U_{13}$ e $U R_{15}$, a correção da umidade relativa das 15:00 para as 13:00 h pode ser usada sem restrições.

\section{REFERÊNCIAS}

BATISTA, A. C. Incêndios florestais. Recife: UFRPE,. 115p. 1990.

CHENEY, N. P. Predicting fire behavior with fire danger tables. Australian Forestry, v.32, n.2, p.71-79. 1968.

DRAPER, N.; SMITH, H. Applied regression analysis. New York, J. Willey, 1981. 725p.

FREESE, F. Elementary statistical methods for foresters. U.S. Department of Agriculture, 87p. 1969

HEIKKILÄ, T. V.; GRONOVIST, R.; JURVÉLIUS, M. Handbook on Forest Fire Control. Helsinki, Forestry Training Programme, 1993. 239p. (Publication 21).

LOWE, J. D. Wildland firefighting practices. Thomson Learning, 2001. 348p

NUNES, J. R. S. Análise do volume e porcentagem de casca em povoamentos de eucaliptos de origem híbrida, segundo a idade, local, espécie e método de regeneração. Viçosa, MG, 1981. Dissertação (Mestrado em Ciência Florestal) - Universidade Federal de Viçosa.

NUNES, J. R. S. FMA ${ }^{+}$- Um novo índice de perigo de incêndios florestais para o estado do Paraná Brasil. Curitiba, 2005. 150p. Tese (Doutorado em Engenharia Florestal) - Setor de Ciências Agrárias, Universidade Federal do Paraná.

SCHROEDER, M. J.; BUCK, C. C. Fire weather. USDA Forest Service, 1970. 229p. (Agriculture Handbook 360.)

SOARES, R. V. Índices de perigo de incêndio. Revista Floresta, Curitiba, v.3, n.3, p.19-40, 1972. 1972 a. 
SOARES, R. V. Determinação de um índice de perigo de incêndio para a região centro paranaense, Brasil. Turrialba, Costa Rica, 72p. (Tese de Mestrado). 1972b.

SOARES, R. V. Incêndios Florestais: controle e uso do fogo. Curitiba: FUPEF, 1985. 213p.

SOARES, R. V. Prevenção e controle de incêndios florestais. ABEAS,. 1984. 120p.

TREWARTHA, G. T.; HORN, L. H., Köppen's classification of climates. In: An Introduction to climate. New York: McGraw-Hill, 1980. p.397-403. 